\title{
Solid-phase microextraction for headspace analysis of key volatile compounds in orange beverage emulsion
}

\begin{abstract}
Headspace solid-phase microextraction (HS-SPME) gas chromatography was used to analyze target flavor compounds in orange beverage emulsion. The effects of SPME fiber (PDMS $100 \mathrm{~lm}$, CAR/PDMS $75 \mathrm{~lm}$, PDMS/DVB $65 \mathrm{~lm}$ and DVB/CAR/PDMS 50/30 lm), adsorption temperature (25-45 C), adsorption time (5-25 min), sample concentration (1-100\%), sample amount (5-12.5 g), pH (2.5-9.5), salt type (K2CO3, Na2CO3, $\mathrm{NaCl}$ and $\mathrm{Na} 2 \mathrm{SO} 4)$, salt amounts $(0-30 \%)$ and stirring mode were studied to develop HS-SPME condition for obtaining the highest extraction efficiency and aroma recovery. For the head space volatile extraction, the optimum conditions were: CAR/PDMS fiber, adsorption at $45 \mathrm{C}$ for $15 \mathrm{~min}, 5$ $\mathrm{g}$ of diluted beverage emulsion (1:100), 15\% (w/w) of $\mathrm{NaCl}$ with stirring and original $\mathrm{pH} 4$. The main volatile flavor compounds were: limonene, $94.9 \%$; myrcene, $1.2 \%$; ethyl butyrate, $1.1 \%$; c-terpinene, $0.41 \%$; linalool, $0.36 \%$; 3-carene, $0.16 \%$; decanal, $0.12 \%$; ethyl acetate, $0.1 \%$; 1-octanol, $0.06 \%$; geranial, $0.05 \%$; b-pinene, $0.04 \%$; octanal, $0.03 \%$; a-pinene, $0.03 \%$; and neral, $0.03 \%$. The linearity was very good in the considered concentration ranges (R2 P0.97). Average recoveries ranged from $88.3 \%$ to $121.7 \%$ and showed good accuracy for the proposed analytical method. Average relative standard deviation (RSD) for five replicate analyses was found to be less than $14 \%$. The limit of detection (LOD) ranged from 0.06 to $2.27 \mathrm{mg} / \mathrm{l}$ for all volatile flavor compounds and confirmed the feasibility of the HS-SPME technique for headspace analysis of orange beverage emulsion. The method was successfully applied for headspace analysis of five commercial orange beverage emulsions.
\end{abstract}

Keyword: Solid-phase microextraction, Gas chromatography, Flavor compounds, Orange beverage emulsion, Extraction efficiency, Headspace analysis 